\title{
Overview of Dark Matter searches at the ATLAS experiment
}

\author{
Bernhard Meirose \\ On behalf of the ATLAS Collaboration \\ Department of Physics, University of Texas at Dallas, $800 \mathrm{~W}$ Campbell Rd, Richardson \\ Dallas, TX 75080, United States of America \\ bernhard.meirose@utdallas.edu
}

Received Day Month 2016

Revised Day Month 2016

Published Day Month 2016

\begin{abstract}
After a brief introduction a concise summary of some of ATLAS experiment's stragies to search for dark matter is presented. Emphasis is given to searches in channels involving the Higgs boson.

Keywords: Dark Matter; Higgs boson; LHC; ATLAS.
\end{abstract}

PACS numbers:14.80.Bn, 12.60.Fr, 14.80.Ec, 95.35.+d

\section{Introduction}

A non-baryonic dark matter (DM) component in the universe is commonly used to explain a range of astrophysical measurements (see, for example, Ref. 1 for a review). Since none of the known Standard Model (SM) particles are adequate DM candidates, new particle types have been hypothesized. Weakly interacting massive particles (WIMPs) are one such class of particle candidates that might be detectable by LHC experiments. They are expected to couple to SM particles through a generic weak interaction, which could be the weak interaction of the SM or a new type of interaction. Such a new particle could account for the correct relic density values for non-relativistic matter in the early universe ${ }^{2}$ as measured by the Planck ${ }^{3}$ and WMAP $^{4}$ satellites if its mass were between a few $\mathrm{GeV}$ and a $\mathrm{TeV}$ and if it had electroweak-scale interaction cross sections. Other new particle physics models such as Supersymmetry also predict WIMPs.

Since the discovery of a Higgs boson with a mass of approximately $125 \mathrm{GeV}^{5,6}$, the properties of this new particle have been studied extensively. All results obtained so far are consistent with the expectations of the long-sought SM Higgs boson. Sizeable deviations from the SM expectation cannot, however, be yet excluded; the total

This is an Open Access article published by World Scientific Publishing Company. It is distributed under the terms of the Creative Commons Attribution 3.0 (CC-BY) License. Further distribution of this work is permitted, provided the original work is properly cited. 
branching ratio of beyond-the-SM decays of the Higgs boson is only weakly constrained, and its value could be as high as $\sim 40 \%^{7}$. In the so-called Higgs-portal models ${ }^{8}$ the Higgs boson can decay to a pair of WIMP dark-matter particles if the decay is kinematically allowed. Decays to WIMPS are generally "invisible" to detectors, because WIMPs interact so weakly. They do not deposit energy in the calorimeter, hence their production leads to signatures with missing transverse momentum $\left(\vec{p}_{\mathrm{T}}^{\text {miss }}\right)$, the magnitude of which is called $E_{\mathrm{T}}^{\text {miss }}$. Collider data can be used to directly constrain the branching ratio of the Higgs boson to invisible particles. Similarly, limits can be placed on the cross section times branching ratio of any additional Higgs bosons decaying predominantly to invisible particles.

\section{Invisible decays of a Higgs boson using vector-boson fusion}

The ATLAS Collaboration searches for invisible decays of a Higgs boson produced via the vector-boson fusion (VBF) (see Ref. 9 and references therein) process as seen in Fig. 1. In the SM, the process $H \rightarrow Z Z \rightarrow 4 \nu$ is an invisible decay of the Higgs boson, but the branching fraction $(\mathrm{BF})$ is $0.1 \%^{10}$, which is below the sensitivity of the search performed by ATLAS. The signature of the VBF process is two jets with a large separation in pseudorapidity and large $E_{\mathrm{T}}^{\text {miss }}$. The observed data rate ${ }^{a}$ is consistent with background estimates. Assuming the SM production cross section, acceptance and efficiency for invisible decays of a Higgs boson with a mass of $125 \mathrm{GeV}$, an upper bound is set on the $\mathrm{BF}(H \rightarrow$ invisible $)<0.28$ at the $95 \%$ confidence level (CL). The results are interpreted in the Higgs-portal darkmatter model where the $90 \%$ CL limit on the $\mathrm{BF}(H \rightarrow$ invisible) leads to an upper bound on the DM-nucleon scattering cross section as a function of the dark-matter particle mass, which is presented Fig. 2. These ATLAS limits are complementary to the results from direct dark-matter detection experiments.

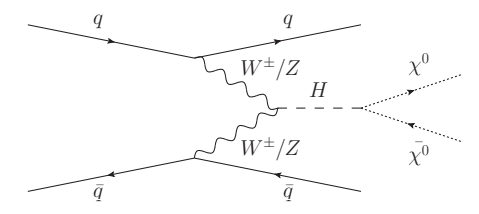

Fig. 1. Feynman diagram for the VBF $H(\rightarrow$ invisible $)$ process from Ref. 9.

\footnotetext{
${ }^{\text {a } U n l e s s ~ o t h e r w i s e ~ s p e c i f i e d, ~ a l l ~ s e a r c h e s ~ s u m m a r i z e d ~ i n ~ t h i s ~ p a p e r ~ w e r e ~ p e r f o r m e d ~ w i t h ~ a ~ d a t a s e t ~}$ corresponding to an integrated luminosity of $20.3 \mathrm{fb}^{-1}$ of proton-proton collisions at $\sqrt{s}=8 \mathrm{TeV}$, which were recorded by the ATLAS detector at the LHC ${ }^{11}$.
} 


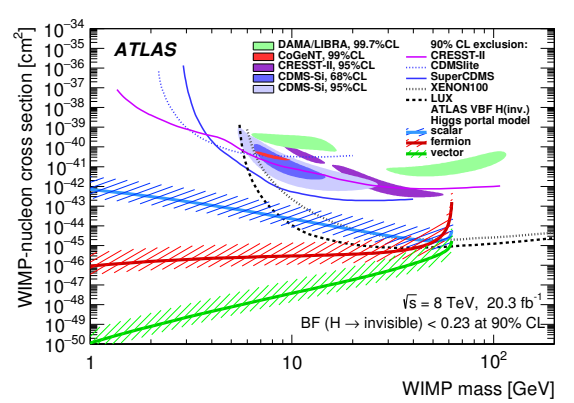

Fig. 2. The WIMP-nucleon cross section as a function of the WIMP mass. The exclusion limits and signals observed by the direct detection experiments are compared to the ATLAS results from the $\mathrm{BF}(H \rightarrow$ invisible $)$ limit in the Higgs-portal scenario, translated into the WIMP-nucleon cross section. The exclusion limits are shown at 90\% CL. Figure from Ref. 9.

\section{Invisible decays of the Higgs in association with a hadronically decaying vector boson}

ATLAS searches for Higgs boson decays to particles that are invisible to the detector in the process where a Higgs boson is produced in association with a vector boson $(V=W$ or $Z)$ that decays hadronically. This results in events with two or more jets and large missing transverse momentum (see Ref. 12 and references therein). No excess of candidates is observed over the expected background. The null results are used to set 95\% CL upper limits on the product of the $V H$ cross sections and the $V \rightarrow j j$ and $H \rightarrow$ inv. decay branching ratio, $\sigma_{V H} \times \mathrm{BR}(H \rightarrow$ inv. $)$, as a function of the Higgs boson mass in the range $115<m_{H}<300 \mathrm{GeV}$ as shown in Fig. 3. The observed upper limit on $\sigma_{V H} \times \mathrm{BR}(H \rightarrow$ inv. $)$ varies from $1.6 \mathrm{pb}$ at a Higgs mass of $115 \mathrm{GeV}$ to $0.13 \mathrm{pb}$ at $300 \mathrm{GeV}$. Assuming SM production and including the $g g \rightarrow H$ contribution as signal the results lead to an observed upper limit of $78 \%$ at the $95 \%$ CL on the branching ratio of a $125 \mathrm{GeV}$ Higss boson to invisible particles.

\section{Invisible decays of the Higgs in association with a $\mathrm{Z}$ Boson}

The ATLAS Collaboration searches for evidence of invisible-particle decay modes of a Higgs boson produced in association with a $Z$ boson (see Ref. 13 and references therein). The search uses $4.5 \mathrm{fb}^{-1}$ of data recorded with the ATLAS detector in 2011 at $\sqrt{s}=7 \mathrm{TeV}$ and $20.3 \mathrm{fb}^{-1}$ of data recorded in 2012 at $\sqrt{s}=8 \mathrm{TeV}$. No deviation from the SM expectation is observed. Assuming the SM rate for $Z H$ production, an upper limit of $75 \%$ at the $95 \%$ CL is set on the branching ratio to invisible-particle decay modes of the Higgs boson at a mass of $125.5 \mathrm{GeV}$. The limit on $\mathrm{BR}(H \rightarrow$ inv. $)$ for the $125.5 \mathrm{GeV}$ Higgs boson is also interpreted in terms of an upper limit on the DM-nucleon scattering cross section. Fig. 4 shows $90 \%$ CL upper limits on the DM-nucleon scattering cross section for three model variants in which 


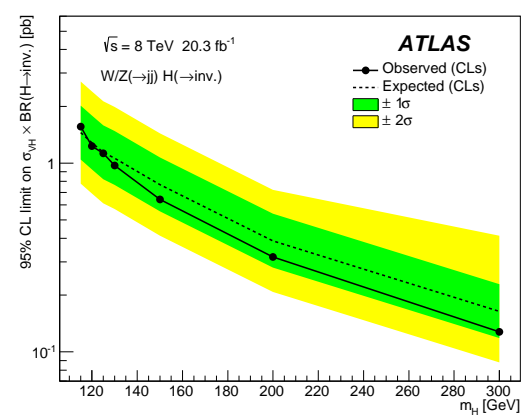

Fig. 3. Upper limits on $\sigma_{V H} \times \mathrm{BR}(H \rightarrow$ inv. $)$ at $95 \%$ CL for a Higgs boson with $115<m_{H}<$ $300 \mathrm{GeV}$. The full and dashed lines show the observed and expected limits, respectively. Figure from Ref. 12.

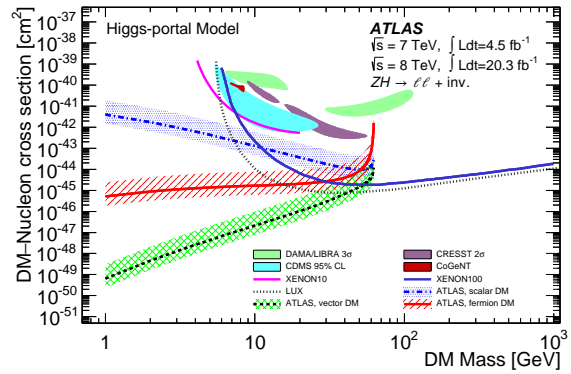

Fig. 4. Limits on the DM-nucleon scattering cross section at 90\% CL, extracted from the $\mathrm{BR}(H \rightarrow$ inv. $)$ limit in a Higgs-portal scenario, compared to results from direct-search experiments. Cross-section limits and favored regions correspond to a $90 \% \mathrm{CL}$, unless stated otherwise in the legend. The results from the direct-search experiments do not depend on the assumptions of the Higgs-portal scenario. Figure from Ref. 13.

a single DM candidate is either a scalar, a vector or a Majorana fermion. Spinindependent results from direct-search experiments are also shown. Direct search results do not depend on the assumptions of the Higgs-portal scenario. Within the constraints of a of a Higgs-portal scenario however, these ATLAS results provide the strongest available limits on low-mass DM candidates. There is no sensitivity to these models once the mass of the DM candidate exceeds half of the SM Higgs boson's mass.

Limits are set on an additional neutral Higgs boson with a mass between $100 \mathrm{GeV}$ and $400 \mathrm{GeV}$ that is produced in in association with a $Z$ boson and decaying to invisible particles. Fig. 5 shows the $95 \%$ CL upper limits on $\sigma_{Z H} \times \mathrm{BR}(H \rightarrow$ inv. $)$ in the mass range $110<m_{H}<400 \mathrm{GeV}$ for the combined 7 and $8 \mathrm{TeV}$ data. 


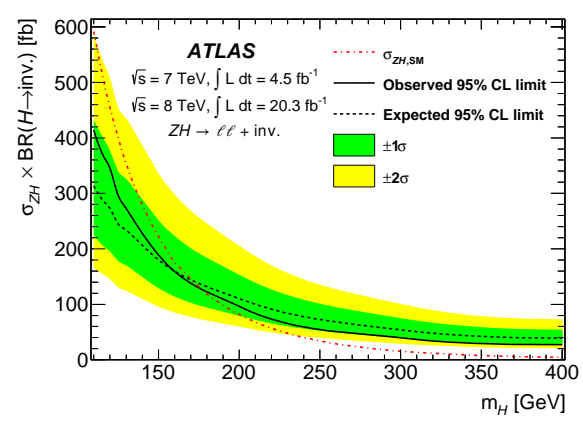

Fig. 5. Upper limits on $\sigma_{Z H} \times \mathrm{BR}(H \rightarrow$ inv. $)$ at $95 \% \mathrm{CL}$ for a Higgs boson with $110<m_{H}<$ $400 \mathrm{GeV}$, for the combined 7 and $8 \mathrm{TeV}$ data. The full and dashed lines show the observed and expected limits, respectively. Figure from Ref. 13.

The expectation for a Higgs boson with a production cross section equal to that expected for a SM Higgs boson and $\operatorname{BR}(H \rightarrow$ inv. $)=1$ is also shown.

\section{Dark matter in events with a monojet plus missing transverse energy}

Events with an energetic jet and large missing transverse momentum in the final state constitute a clean and distinctive "monojet" signature in searches for new physics beyond the SM at colliders. ATLAS uses monojet-like signatures in the search for WIMPs (see Ref. 14 and references therein). Here, WIMPs are assumed to be produced in pairs, and events are identified via the presence of an energetic jet from initial-state radiation (ISR). The interaction of WIMPs with SM particles is described as a contact interaction using an effective field theory (EFT) approach, mediated by a new heavy particle or particles having a mass that is too large to be produced directly at the LHC (see Fig. 6(a)). The validity of the effective field assumption depends on the momentum transfer of the process modeled, which should be below the energy scale of the underlying interactions. Besides the EFT operators, the pair production of WIMPs is investigated within a so-called simplified model, where a pair of WIMPs couples to a pair of quarks explicitly via a new $Z^{\prime}$ vector boson mediator particle (see Fig. 6(b)) .

Monojet plus $E_{\mathrm{T}}^{\text {miss }}$ candidates are selected by requiring at least one jet with transverse momentum $p_{\mathrm{T}}>120 \mathrm{GeV}$ and no leptons. Nine signal regions are considered with increasing missing transverse momentum requirements between $E_{\mathrm{T}}^{\text {miss }}>150 \mathrm{GeV}$ and $E_{\mathrm{T}}^{\text {miss }}>700 \mathrm{GeV}$. Good agreement is observed between the number of observed events and SM expectations. The results are presented as exclusion limits on pair production of weakly interacting dark matter candidates. Figure 7 shows the upper limits for the WIMP-nucleon scattering and WIMP annihilation cross sections. The result depends strongly on the EFT operator and the 


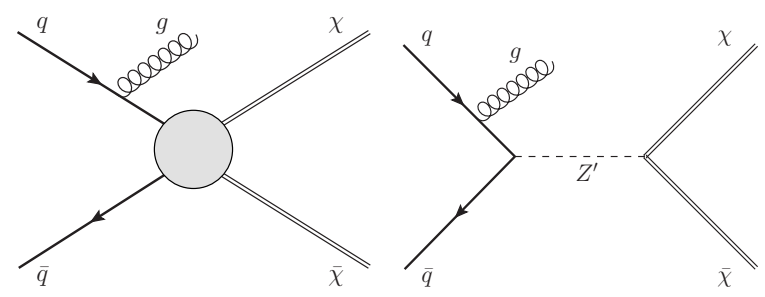

Fig. 6. Feynman diagrams for the production of weakly interacting massive particle pairs $\chi \bar{\chi}$ associated with a jet from initial-state radiation of a gluon, $g$. (a) A contact interaction described with effective operators. (b) A simplified model with a $Z^{\prime}$ boson. Figures from Ref. 14.

values for the couplings considered. The limits remain generally valid for WIMP masses up to $O(100) \mathrm{GeV}$.
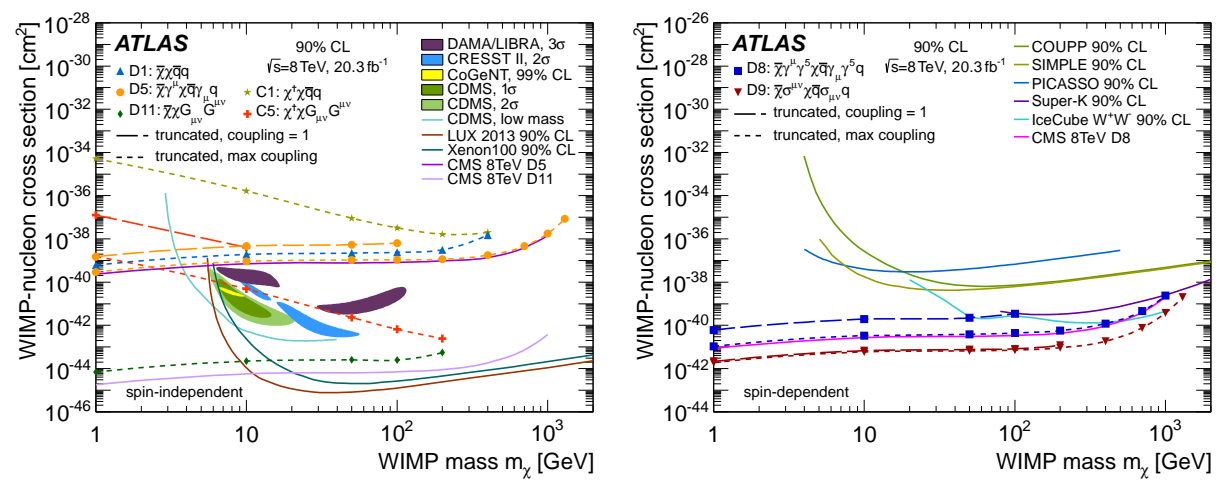

Fig. 7. Inferred $90 \%$ CL limits on (a) the spin-independent and (b) spin-dependent WIMPnucleon scattering cross section as a function of DM mass $m_{\chi}$ for different operators. Results from direct-detection experiments for the spin-independent and spin-dependent cross section, and the CMS results are shown for comparison. Figures from Ref. 14.

\section{Dark matter in events with heavy quarks and missing transverse momentum}

ATLAS searches for dark matter pair production in association with bottom or top quarks (see Ref. 15 and references therein). Events with large missing transverse momentum are selected when produced in association with high-momentum jets, one or more of which are identified as jets containing $b$-quarks. The dominant Feynman diagrams for these processes are shown in Fig. 8.

Candidate events with top quarks are selected by requiring a high jet multiplicity and in some cases a single lepton. To search for these processes, selection criteria are defined to reconstruct the various production and decay modes of these heavy-quark 


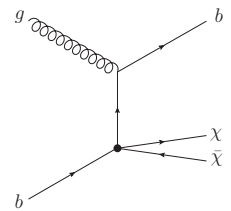

(a)

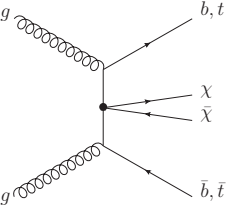

(b)

Fig. 8. Dominant Feynman diagrams for DM production in conjunction with (a) a single $b$-quark and (b) a heavy quark (bottom or top) pair using an effective field theory approach. Figure from Ref. 15 .

final states. The data are consistent with the SM expectations, allow limits to be set on the $\chi$-nucleon cross-section. Fig. 9 show the corresponding $90 \%$ CL exclusion curves for the spin-independent (left) and spin-dependent (right) $\chi$-nucleon crosssection for EFT operators as a function of $m_{\chi}$. The most stringent limits set by direct detection experiments are also shown. Limits are especially strong in the low-mass region.
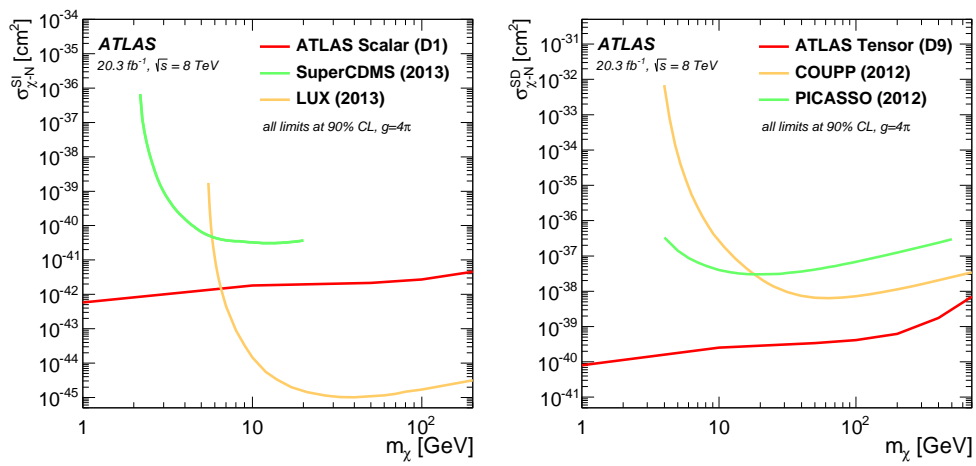

Fig. 9. Upper limits at $90 \% \mathrm{CL}$ on the spin-independent $\chi$-nucleon cross-section $\left(\sigma_{\chi-\mathrm{N}}^{\mathrm{SI}}\right)$ for the EFT operator D1 (left) and on the spin-dependent $\chi$-nucleon cross-section $\left(\sigma_{\chi-\mathrm{N}}^{\mathrm{SD}}\right)$ for the EFT operator D9 (right) as a function of $m_{\chi}$. The yellow and green curves represent the exclusion limits set by direct detection experiments. Figure from Ref. 15.

\section{Summary, other searches and preliminary Run 2 prospects}

An overview of the ATLAS experiment's DM searches is presented. None of the searches find deviations from the SM, and are presented as upper limits. Additional searches not described in this paper (Refs.: 16, 17, 18, 19, 20 and 21) find no deviations from the SM. The anticipated ATLAS sensitivity to DM channels with the full Run 2 data set can be found in Ref. 22 . 


\section{Acknowledgments}

This work has been supported by US DOE Grant Number DE-SC0010384 and by the organizers of CosPA2015.

\section{References}

1. G. Bertone, D. Hooper and J. Silk, Phys. Rept. 405, 279 (2005).

2. E. W. Kolb and M. S. Turner, Front. Phys. 69, 1 (1990).

3. P. A. R. Ade et al., Astron. Astrophys. 571, p. A16 (2014).

4. G. Hinshaw et al., Astrophys. J. Suppl. 208, p. 19 (2013).

5. ATLAS Collaboration, Phys. Lett. B716, 1 (2012).

6. CMS Collaboration, Phys. Lett. B716, 30 (2012).

7. CMS Collaboration, Eur. Phys. J. C75, p. 212 (2015).

8. B. Patt and F. Wilczek, MIT-CTP-3745 (2006).

9. ATLAS Collaboration, CERN-PH-EP-2015-186 (2015).

10. S. Dittmaier et al., CERN-2011-002 (2011).

11. ATLAS Collaboration, JINST 3, p. S08003 (2008).

12. ATLAS Collaboration, Eur. Phys. J. C75, p. 337 (2015).

13. ATLAS Collaboration, Phys. Rev. Lett. 112, p. 201802 (2014).

14. ATLAS Collaboration, Eur. Phys. J. C75, p. 299 (2015), [Erratum: Eur. Phys. J.C75,no.9,408(2015)].

15. ATLAS Collaboration, Eur. Phys. J. C75, p. 92 (2015).

16. ATLAS Collaboration, Phys. Rev. Lett. 112, p. 041802 (2014).

17. ATLAS Collaboration, JHEP 09, p. 037 (2014).

18. ATLAS Collaboration, Phys. Rev. D90, p. 012004 (2014).

19. ATLAS Collaboration, Phys. Rev. D91, p. 012008 (2015), [Erratum: Phys. Rev.D92,no.5,059903(2015)].

20. ATLAS Collaboration, Phys. Rev. Lett. 115, p. 131801 (2015).

21. ATLAS Collaboration, CERN-PH-EP-2015-247 (2015).

22. ATLAS Collaboration, ATL-PHYS-PUB-2014-007 (2014). 